\title{
Bargmann Symmetry Constraint and Binary Nonlinearization of Super NLS-MKdV Hierarchy
}

\author{
Sixing Tao ${ }^{1^{*}}$, Hui Shi ${ }^{2}$ \\ ${ }^{1}$ School of Mathematics and Information Science, Shangqiu Normal University, Shangqiu, China \\ ${ }^{2}$ School of Physics and Electronic Information, Shangqiu Normal University, Shangqiu, China \\ Email: " taosixing@163.com
}

Received 2013

\begin{abstract}
An explicit Bargmann symmetry constraint is computed and its associated binary nonlinearization of Lax pairs is carried out for the super NLS-MKdV hierarchy. Under the obtained symmetry constraint, the $n$-th flow of the super NLS-MKdV hierarchy is decomposed into two super finite-dimensional integrable Hamiltonian systems, defined over the super-symmetry manifold $\mathbb{R}^{4 N 2 N}$ with the corresponding dynamical variables $x$ and $t_{n}$. The integrals of motion required for Liouville integrability are explicitly given.
\end{abstract}

Keywords: Symmetry Constraints; Binary Nonlinearization; Super NLS-MKdV Hierarchy; Super Finite Dimensional Integrable Hamiltonian Systems

\section{Introduction}

For almost twenty years, much attention has been paid to the construction of finite-dimensional integrable systems from soliton equations by using symmetry constraints. Either (2+1)-dimensional soliton equations [1,2] or $(1+$ 1)-dimensional soliton equations $[3,4]$ can be decomposed into compatible finite-dimensional integrable systems. It is known that a crucial idea in carrying out symmetry constraints is the nonlinearization of Lax pairs for soliton hierarchies. The nonlinearization of Lax pairs is classified into mono-nonlinearization [5,6] and binary nonlinearization $[7,8]$.

The technique of nonlinearization has been successfully applied to many well-known (1+1)-dimensional soliton equations, such as the AKNS hierarchy [3], the KdV hierarchy [4] and the Dirac hierarchy [9]. But there are few results on nonlinearization of super integrable systems, existing in the literature. Very recently, nonlinearization were made for the super AKNS hierarchy, the super Dirac hierarchy and their corresponding super finite-dimensional hierarchies were generated in Refs. [10-12]. Dong presented the super Hamiltonian structures of the super NLS-MKdV hierarchy [13]. In this paper, we would like to consider the binary nonlinearization of the super NLS-MKdV hierarchy.

This paper is organized as follows. In the next section, we will recall the super NLS-MKdV soliton hierarchy and its super Hamiltonian structure. Then in Section 3,

*Corresponding author. we compute a Bargmann symmetry constraint for the potential of the super NLS-MKdV hierarchy. In Section 4, we apply binary nonlinearization method to the super NLS-MKdV hierarchy, and then obtain super finite dimensional integrable Hamiltonian hierarchy on the super symmetry manifold $\mathbb{R}^{4 N 2 N}$, whose integrals of motion are explicitly given.

\section{The Super NLS-MKdV Hierarchy}

The super NLS-MKdV spectral problem associated with the Lie super algebra $B(0,1)$ is given by

$$
\begin{aligned}
& \phi_{x}=U \phi, U=\left(\begin{array}{ccc}
\frac{1}{2} \lambda & \frac{1}{2}(q+r) & \alpha \\
\frac{1}{2}(q-r) & -\frac{1}{2} \lambda & \beta \\
\beta & -\alpha & 0
\end{array}\right), \\
& u=\left(\begin{array}{c}
q \\
r \\
\alpha \\
\beta
\end{array}\right), \phi=\left(\begin{array}{l}
\phi_{1} \\
\phi_{2} \\
\phi_{3}
\end{array}\right),
\end{aligned}
$$

where $\lambda$ is a spectral parameter, $q$ and $r$ are even variables, $\alpha$ and $\beta$ are odd variables[14]. Taking

$$
V=\left(\begin{array}{ccc}
\frac{1}{2} A & B & \rho \\
C & -\frac{1}{2} A & \delta \\
\delta & -\rho & 0
\end{array}\right),
$$

the co-adjoint equation associated with Equation (1) $V_{x}=[U, V]$ gives 


$$
\left\{\begin{array}{c}
A_{x}=(-q+r) B+(q+r) C+2 \beta \rho+2 \alpha \delta, \\
B_{x}=\lambda B-\frac{1}{2}(q+r) A-2 \alpha \rho, \\
C_{x}=-\lambda C+\frac{1}{2}(q-r) A+2 \beta \delta, \\
2 \rho_{x}=\lambda \rho-\alpha A-2 \beta B+(q+r) \delta, \\
2 \delta_{x}=-\lambda \delta+\beta A-2 \alpha C+(q-r) \rho .
\end{array}\right.
$$

If we set

$$
\begin{aligned}
& A=\sum_{i \geq 0} A_{i} \lambda^{-i}, B=\sum_{i \geq 0} B_{i} \lambda^{-i}, C=\sum_{i \geq 0} C_{i} \lambda^{-i}, \\
& \rho=\sum_{i \geq 0} \rho_{i} \lambda^{-i}, \delta=\sum_{i \geq 0} \delta_{i} \lambda^{-i},
\end{aligned}
$$

then Equation (2) is equivalent to

$$
\left\{\begin{array}{c}
B_{i+1}=\frac{1}{2}(q+r) A_{i}+B_{i, x}+2 \alpha \rho_{i}, \\
C_{i+1}=\frac{1}{2}(q-r) A_{i}-C_{i, x}+2 \beta \delta_{i}, \\
\rho_{i+1}=\alpha A_{i}+2 \beta B_{i}+2 \rho_{i, x}-(q+r) \delta_{i}, \\
\delta_{i+1}=\beta A_{i}-2 \alpha C_{i}+(q-r) \rho_{i}-2 \delta_{i, x}, \\
A_{i+1, x}=(-q+r) B_{i+1}+(q+r) C_{i+1}+2 \beta \rho_{i+1}+2 \alpha \delta_{i+1}, i \geq 0 .
\end{array}\right.
$$

which results in the recurrence relations

$$
\left\{\begin{array}{c}
\left(B_{i+1}+C_{i+1}, C_{i+1}-B_{i+1}, 4 \delta_{i+1},-4 \rho_{i+1}\right)^{\mathrm{T}} \\
=L\left(B_{i}+C_{i}, C_{i}-B_{i}, 4 \delta_{i},-4 \rho_{i}\right)^{\mathrm{T}}, \\
A_{i}=\partial^{-1}\left(q\left(C_{i}-B_{i}\right)+r\left(B_{i}+C_{i}\right)+2 \beta \rho_{i}+2 \alpha \delta_{i}\right), i \geq 0 .
\end{array}\right.
$$

where

Upon choosing the initial conditions

$$
B_{0}=C_{0}=\rho_{0}=\delta_{0}=0, A_{0}=1,
$$

all other $A_{i}, B_{i}, C_{i}, \rho_{i}, \delta_{i}(i \geq 1)$ can be worked out by the recurrence relations Equation (5). The first few sets are:

$$
\begin{aligned}
A_{1}= & 0, B_{1}=\frac{1}{2}(q+r), C_{1}=\frac{1}{2}(q-r), \\
\rho_{1}= & \alpha, \delta_{1}=\beta, A_{2}=-\frac{1}{2} q^{2}+\frac{1}{2} r^{2}-4 \alpha \beta, \\
B_{2}= & \frac{1}{2} q_{x}+\frac{1}{2} r_{x}, C_{2}=-\frac{1}{2} q_{x}+\frac{1}{2} r_{x}, \\
\rho_{2}= & 2 \alpha_{x}, \delta_{2}=-2 \beta_{x}, \\
B_{3}= & \frac{1}{2} q_{x x}+\frac{1}{2} r_{x x}+4 \alpha \alpha_{x}-\frac{1}{4} q^{3}-\frac{1}{4} q^{2} r+\frac{1}{4} q r^{2} \\
& +\frac{1}{4} r^{3}-2 q \alpha \beta-2 r \alpha \beta, \\
C_{3}= & \frac{1}{2} q_{x x}-\frac{1}{2} r_{x x}-4 \beta \beta_{x}-\frac{1}{4} q^{3}+\frac{1}{4} q^{2} r+\frac{1}{4} q r^{2} \\
& -\frac{1}{4} r^{3}-2 q \alpha \beta+2 r \alpha \beta, \\
\rho_{3}= & 4 \alpha_{x x}+q_{x} \beta+r_{x} \beta+2 q \beta_{x}+2 r \beta_{x}-\frac{1}{2} q^{2} \alpha+\frac{1}{2} r^{2} \alpha, \\
\delta_{3}= & 4 \beta_{x x}+q_{x} \alpha-r_{x} \alpha+2 q \alpha_{x}-2 r \alpha_{x}-\frac{1}{2} q^{2} \beta+\frac{1}{2} r^{2} \beta,
\end{aligned}
$$

$$
A_{3}=q_{x} r-q r_{x}-8 \alpha_{x} \beta+8 \alpha \beta_{x} .
$$

Let us associate the spectral problem Equation (1) with the following auxiliary problem

$$
\phi_{t_{n}}=V^{(n)} \phi=\left(\lambda^{n} V\right)_{+} \phi
$$

with

$$
V^{(n)}=\sum_{i=0}^{n}\left(\begin{array}{ccc}
\frac{1}{2} A_{i} & B_{i} & \rho_{i} \\
C_{i} & -\frac{1}{2} A_{i} & \delta_{i} \\
\delta_{i} & -\rho_{i} & 0
\end{array}\right) \lambda^{n-i},
$$

where the symbol " + " denotes taking the non-negative part in the power of $\lambda$.

The compatible conditions of the spectral problem Equation (1) and the auxiliary problem Equation (7) are

$$
U_{t_{n}}-V_{x}^{(n)}+\left[U, V^{(n)}\right]=0,
$$

which infer the super NLS-MKdV hierarchy

$$
\begin{aligned}
& u_{t_{n}}=K_{n}=\left(B_{n+1}-C_{n+1}, B_{n+1}+C_{n+1}, \frac{1}{2} \rho_{n+1},-\frac{1}{2} \delta_{n+1}\right)^{\mathrm{T}}, \\
& n \geq 0 .
\end{aligned}
$$

Here $u_{t_{n}}=K_{n}$ in Equation (9) is called the $n$-th NLS - MKdV flow of this hierarchy.

Using the super trace identity

$$
\frac{\delta}{\delta u} \int \operatorname{Str}\left(V \frac{\partial U}{\partial \lambda}\right) \mathrm{d} x=\left(\lambda^{-\gamma} \frac{\partial}{\partial \lambda} \lambda^{\gamma}\right) \operatorname{Str}\left(\frac{\partial U}{\partial u} V\right),
$$

where Str means the super trace [14,15], we have

$$
\left(\begin{array}{c}
B_{i+1}+C_{i+1} \\
C_{i+1}-B_{i+1} \\
4 \delta_{i+1} \\
-4 \rho_{i+1}
\end{array}\right)=\frac{\delta}{\delta u} H_{i}, H_{i}=\int-\frac{A_{i+2}}{i+1} \mathrm{~d} x, i \geq 0
$$

Therefore, the super NLS-MKdV soliton hierarchy Equation (9) can be written as the following super Hamiltonian form:

$$
u_{t_{n}}=J \frac{\delta H_{n}}{\delta u}
$$

where

$$
J=\left(\begin{array}{cccc}
0 & -1 & 0 & 0 \\
1 & 0 & 0 & 0 \\
0 & 0 & 0 & -\frac{1}{8} \\
0 & 0 & -\frac{1}{8} & 0
\end{array}\right)
$$

is a super symplectic operator, and $H_{n}$ is given by Equation (11).

$$
L=\left(\begin{array}{cccc}
q \partial^{-1} r & -\partial+q \partial^{-1} q & \frac{1}{2}\left(\beta+q \partial^{-1} \alpha\right) & -\frac{1}{2}\left(\alpha+q \partial^{-1} \beta\right) \\
-\partial-r \partial^{-1} r & -r \partial^{-1} q & \frac{1}{2}\left(\beta-r \partial^{-1} \alpha\right) & \frac{1}{2}\left(\alpha+r \partial^{-1} \beta\right) \\
-4 \alpha+4 \beta \partial^{-1} r & -4 \alpha+4 \beta \partial^{-1} q & -2 \partial+2 \beta \partial^{-1} \alpha & -2 \beta \partial^{-1} \beta-q+r \\
-4 \beta-4 \alpha \partial^{-1} r & 4 \beta-4 \alpha \partial^{-1} q & -2 \alpha \partial^{-1} \alpha+q+r & 2 \partial+2 \alpha \partial^{-1} \beta
\end{array}\right) .
$$


The first non-trivial nonlinear equation of the super NLS-MKdV hierarcy (9) is given by its second flow

$$
\left\{\begin{array}{c}
q_{t_{2}}=r_{x x}+4 \alpha \alpha_{x}+4 \beta \beta_{x}-\frac{1}{2} q^{2} r+\frac{1}{2} r^{3}-4 r \alpha \beta, \\
r_{t_{2}}=q_{x x}+4 \alpha \alpha_{x}-4 \beta \beta_{x}-\frac{1}{2} q^{3}+\frac{1}{2} q r^{2}-4 q \alpha \beta, \\
\alpha_{t_{2}}=2 \alpha_{x x}+\frac{1}{2} q_{x} \beta+\frac{1}{2} r_{x} \beta+q \beta_{x}+r \beta_{x}-\frac{1}{4} q^{2} \alpha+\frac{1}{4} r^{2} \alpha, \\
\beta_{t_{2}}=-2 \beta_{x x}-\frac{1}{2} q_{x} \alpha+\frac{1}{2} r_{x} \alpha-q \alpha_{x}+r \alpha_{x}+\frac{1}{4} q^{2} \beta-\frac{1}{4} r^{2} \beta .
\end{array}\right.
$$

which possesses a Lax pair of $U$ defined in Equation (1) and $V^{(2)}$ defined by

\section{Bargmann Symmetry Constraint of Super NLS-MKdV Hierarchy}

In order to compute a Bargmann symmetry constraint, we consider the following adjoint spectral problem of the spectral problem:

$$
\psi_{x}=-U^{S t} \psi=\left(\begin{array}{ccc}
-\frac{1}{2} \lambda & -\frac{1}{2}(q-r) & \beta \\
-\frac{1}{2}(q+r) & \frac{1}{2} \lambda & -\alpha \\
-\alpha & -\beta & 0
\end{array}\right), \psi=\left(\begin{array}{c}
\psi_{1} \\
\psi_{2} \\
\psi_{3}
\end{array}\right),
$$

where St means the super transposition. The following result is a general formula for the variational derivative with respect to the potential $u$ (see[3] for the classical case).

Lemma 1 [10-12] Let $U(u, \lambda)$ be an even matrix of order $m+n$ depending on $u, u_{x}, u_{x x}, \cdots$, and a parameter $\lambda$. Suppose that $\phi=\left(\phi_{e}, \phi_{o}\right)^{\mathrm{T}}$ and $\psi=\left(\psi_{e}, \psi_{o}\right)^{\mathrm{T}}$ satisfy the spectral problem and the adjoint spectral problem

$$
\phi_{x}=U(u, \lambda) \phi, \psi_{x}=-U^{S t} \psi,
$$

where $\phi_{e}=\left(\phi_{1}, \cdots, \phi_{m}\right)$ and $\psi_{e}=\left(\psi_{1}, \cdots, \psi_{m}\right)$ are even eigenfunctions, and $\phi_{o}=\left(\phi_{m}, \cdots, \phi_{m+n}\right)$ and $\psi_{o}=\left(\psi_{m}, \cdots, \psi_{m+n}\right)$ are odd eigenfunctions. Then the variational derivative of the parameter $\lambda$ with respect to the potential $u$ is given by

$$
\frac{\delta \lambda}{\delta u}=\frac{\left(\psi_{e},(-1)^{p(u)} \psi_{o}\right)\left(\frac{\partial U}{\partial u}\right) \phi}{-\int \psi^{T}\left(\frac{\partial U}{\partial \lambda}\right) \phi \mathrm{d} x},
$$

where we denote

$$
p(v)=\left\{\begin{array}{c}
0, v \text { is an even variable, } \\
1, v \text { is an odd variable. }
\end{array}\right.
$$

By Lemma 1, it is not difficult to find that

$$
\frac{\delta \lambda}{\delta u}=\frac{1}{E}\left(\begin{array}{c}
\frac{1}{2} \psi_{1} \phi_{2}+\frac{1}{2} \psi_{2} \phi_{1} \\
\frac{1}{2} \psi_{1} \phi_{2}-\frac{1}{2} \psi_{2} \phi_{1} \\
\psi_{1} \phi_{3}+\psi_{3} \phi_{2} \\
\psi_{2} \phi_{3}-\psi_{3} \phi_{1}
\end{array}\right)
$$

where $E=-\int \frac{1}{2}\left(\psi_{1} \phi_{1}-\psi_{2} \phi_{2}\right) \mathrm{d} x$. If we consider zero boundary conditions $\lim _{|x| \rightarrow \infty} \phi=\lim _{|x| \rightarrow \infty} \psi=0$, we can obtain a characteristic property- a recurrence relation for the variational derivative of $\lambda$ :

$$
L \frac{\delta \lambda}{\delta u}=\lambda \frac{\delta \lambda}{\delta u},
$$

where $L$ and $\frac{\delta \lambda}{\delta u}$ are given by Equation (4) and Equation (18), respectively.

Let us now discuss the spatial systems:

$$
\left\{\begin{array}{l}
\left(\begin{array}{l}
\phi_{1 j} \\
\phi_{2 j} \\
\phi_{3 j}
\end{array}\right)_{x}=\left(\begin{array}{ccc}
\frac{1}{2} \lambda & \frac{1}{2}(q+r) & \alpha \\
\frac{1}{2}(q-r) & -\frac{1}{2} \lambda & \beta \\
\beta & -\alpha & 0
\end{array}\right)\left(\begin{array}{l}
\phi_{1 j} \\
\phi_{2 j} \\
\phi_{3 j}
\end{array}\right), \\
\left(\begin{array}{l}
\psi_{1 j} \\
\psi_{2 j} \\
\psi_{3 j}
\end{array}\right)_{x}=\left(\begin{array}{ccc}
-\frac{1}{2} \lambda & -\frac{1}{2}(q-r) & \beta \\
-\frac{1}{2}(q+r) & \frac{1}{2} \lambda & -\alpha \\
-\alpha & -\beta & 0
\end{array}\right)\left(\begin{array}{l}
\psi_{1 j} \\
\psi_{2 j} \\
\psi_{3 j}
\end{array}\right),
\end{array}\right.
$$

and the temporal systems:

$$
\begin{gathered}
V^{(2)}=\left(\begin{array}{ccc}
\frac{1}{2} \lambda^{2}-\frac{1}{4} q^{2}+\frac{1}{4} r^{2}-2 \alpha \beta & \frac{1}{2}(q+r) \lambda+\frac{1}{2}\left(q_{x}+r_{x}\right) & \alpha \lambda+2 \alpha_{x} \\
\frac{1}{2}(q-r) \lambda-\frac{1}{2}\left(q_{x}-r_{x}\right) & -\frac{1}{2} \lambda^{2}+\frac{1}{4} q^{2}-\frac{1}{4} r^{2}+2 \alpha \beta & \beta \lambda-2 \beta_{x} \\
\beta \lambda-2 \beta_{x} & -\alpha \lambda-2 \alpha_{x} & 0
\end{array}\right) . \\
\left(\begin{array}{l}
\phi_{1 j} \\
\phi_{2 j} \\
\phi_{3 j}
\end{array}\right)_{t_{n}}=\left(\begin{array}{ccc}
\sum_{i=0}^{n} \frac{1}{2} A_{i} \lambda_{j}^{n-i} & \sum_{i=0}^{n} B_{i} \lambda_{j}^{n-i} & \sum_{i=0}^{n} \rho_{i} \lambda_{j}^{n-i} \\
\sum_{i=0}^{n} C_{i} \lambda_{j}{ }^{n-i} & -\sum_{i=0}^{n} \frac{1}{2} A_{i} \lambda_{j}^{n-i} & \sum_{i=0}^{n} \delta_{i} \lambda_{j}^{n-i} \\
\sum_{i=0}^{n} \delta_{i} \lambda_{j}^{n-i} & -\sum_{i=0}^{n} \rho_{i} \lambda_{j}^{n-i} & 0
\end{array}\right)\left(\begin{array}{l}
\phi_{1 j} \\
\phi_{2 j} \\
\phi_{3 j}
\end{array}\right), \\
\left(\begin{array}{l}
\psi_{1 j} \\
\psi_{2 j} \\
\psi_{3 j}
\end{array}\right)_{t_{n}}=\left(\begin{array}{ccc}
-\sum_{i=0}^{n} \frac{1}{2} A_{i} \lambda_{j}^{n-i} & -\sum_{i=0}^{n} C_{i} \lambda_{j}^{n-i} & \sum_{i=0}^{n} \delta_{i} \lambda_{j}^{n-i} \\
-\sum_{i=0}^{n} B_{i} \lambda_{j}^{n-i} & \sum_{i=0}^{n} \frac{1}{2} A_{i} \lambda_{j}^{n-i} & -\sum_{i=0}^{n} \rho_{i} \lambda_{j}^{n-i} \\
-\sum_{i=0}^{n} \rho_{i} \lambda_{j}^{n-i} & -\sum_{i=0}^{n} \delta_{i} \lambda_{j}^{n-i} & 0
\end{array}\right)\left(\begin{array}{l}
\psi_{1 j} \\
\psi_{2 j} \\
\psi_{3 j}
\end{array}\right),
\end{gathered}
$$


where $1 \leq j \leq N$ and $\lambda_{1}, \lambda_{2}, \cdots, \lambda_{N}$ are $N$ distinct parameters. Now for systems Equation (21) and Equation (22), we have the following symmetry constraints:

$$
\frac{\delta}{\delta u} H_{k}=\sum_{j=1}^{N} \gamma_{j} \frac{\delta \lambda_{j}}{\delta u}, k \geq 0 .
$$

The symmetry constraints in the case of $k=0$ is called a Bargmann constraint (see[8]). If taking $\gamma_{i}=E_{j}$ $=-\int \frac{1}{2}\left(\psi_{1 j} \phi_{1 j}-\psi_{2 j} \phi_{2 j}\right) \mathrm{d} x$, then it leads to an expression for the potential $u$, i.e.

$$
\left\{\begin{array}{c}
q=\frac{1}{2}\left(\left\langle\Psi_{1}, \Phi_{2}\right\rangle+\left\langle\Psi_{2}, \Phi_{1}\right\rangle\right), \\
r=\frac{1}{2}\left(\left\langle\Psi_{2}, \Phi_{1}\right\rangle-\left\langle\Psi_{1}, \Phi_{2}\right\rangle\right), \\
\alpha=-\frac{1}{4}\left(\left\langle\Psi_{2}, \Phi_{3}\right\rangle-\left\langle\Psi_{3}, \Phi_{1}\right\rangle\right), \\
\beta=\frac{1}{4}\left(\left\langle\Psi_{1}, \Phi_{3}\right\rangle+\left\langle\Psi_{3}, \Phi_{2}\right\rangle\right),
\end{array}\right.
$$

where we use the following notation

$$
\Phi_{i}=\left(\Phi_{i 1}, \cdots, \Phi_{i N}\right)^{\mathrm{T}}, \Psi_{i}=\left(\Psi_{i 1}, \cdots, \Psi_{i N}\right)^{\mathrm{T}}, i=1,2,3 .
$$

and $\langle\cdot, \cdot\rangle$ denotes the standard inner product of the Euclidean space $\mathbb{R}^{N}$.

\section{Binary Nonlinearization of Super NLS -MKdV Hierarchy}

In order to perform binary nonlineqrization to the super NLS-MKdV hierarchy. To this end, let us substituting Equation (24) into the Lax pairs and adjoint Lax pairs Equation (21) and Equation (22), and then we obtain the following nonlinearized spatial Lax pairs and adjoint Lax pairs:

$$
\left\{\begin{array}{c}
\left(\begin{array}{l}
\phi_{1 j} \\
\phi_{2 j} \\
\phi_{3 j}
\end{array}\right)_{x}=\left(\begin{array}{ccc}
\frac{1}{2} \lambda & \frac{1}{2}(\tilde{q}+\tilde{r}) & \tilde{\alpha} \\
\frac{1}{2}(\tilde{q}-\tilde{r}) & -\frac{1}{2} \lambda & \tilde{\beta} \\
\tilde{\beta} & -\tilde{\alpha} & 0
\end{array}\right)\left(\begin{array}{l}
\phi_{1 j} \\
\phi_{2 j} \\
\phi_{3 j}
\end{array}\right), \\
\left(\begin{array}{l}
\psi_{1 j} \\
\psi_{2 j} \\
\psi_{3 j}
\end{array}\right)_{x}=\left(\begin{array}{ccc}
-\frac{1}{2} \lambda & -\frac{1}{2}(\tilde{q}-\tilde{r}) & \tilde{\beta} \\
-\frac{1}{2}(\tilde{q}+\tilde{r}) & \frac{1}{2} \lambda & -\tilde{\alpha} \\
-\tilde{\alpha} & -\tilde{\beta} & 0
\end{array}\right)\left(\begin{array}{l}
\psi_{1 j} \\
\psi_{2 j} \\
\psi_{3 j}
\end{array}\right),
\end{array}\right.
$$

and

where $1 \leq j \leq N$ and $\tilde{P}$ means an expression of $P(u)$ under the explicit constraint Equation (24). Note that the spatial part of the nonlinearized system Equation (25) is a system of ordinary differential equations with an independent variables $u$, but for a given $n(n \geq 2)$, the $t_{n}$ part of the nonlinearized system Equation (26) is a system of ordinary differential equations. Obviously, the system Equation (25) can be written as

where $\Lambda=\operatorname{diag}\left(\lambda_{1}, \cdots, \lambda_{N}\right)$. When $n=1$, the system Equation (26) is exactly the system Equation (25) with $t_{1}=x$. The system Equation (25) or Equation (27) can be written as the following super Hamiltonian form:

$$
\left\{\begin{array}{c}
\Phi_{1, x}=\frac{\partial H_{1}}{\partial \Psi_{1}}, \Phi_{2, x}=\frac{\partial H_{1}}{\partial \Psi_{2}}, \Phi_{3, x}=\frac{\partial H_{1}}{\partial \Psi_{3}}, \\
\Psi_{1, x}=-\frac{\partial H_{1}}{\partial \Phi_{1}}, \Psi_{2, x}=-\frac{\partial H_{1}}{\partial \Phi_{2}}, \Psi_{3, x}=\frac{\partial H_{1}}{\partial \Phi_{3}} .
\end{array}\right.
$$

where

$$
\begin{aligned}
H_{1}= & \frac{1}{2}\left\langle\Lambda \Psi_{1}, \Phi_{1}\right\rangle-\frac{1}{2}\left\langle\Lambda \Psi_{2}, \Phi_{2}\right\rangle-\frac{1}{4}\left(\left\langle\Psi_{2}, \Phi_{3}\right\rangle-\left\langle\Psi_{3}, \Phi_{1}\right\rangle\right) \\
& \times\left(\left\langle\Psi_{1}, \Phi_{3}\right\rangle+\left\langle\Psi_{3}, \Phi_{2}\right\rangle\right) .
\end{aligned}
$$

$$
\begin{gathered}
\left\{\begin{array}{c}
\phi_{1 j} \\
\phi_{2 j} \\
\phi_{3 j}
\end{array}\right)_{t_{n}}=\left(\begin{array}{ccc}
\sum_{i=0}^{n} \frac{1}{2} \tilde{A}_{i} \lambda_{j}^{n-i} & \sum_{i=0}^{n} \tilde{B}_{i} \lambda_{j}^{n-i} & \sum_{i=0}^{n} \tilde{\rho}_{i} \lambda_{j}^{n-i} \\
\sum_{i=0}^{n} \tilde{C}_{i} \lambda_{j}^{n-i} & -\sum_{i=0}^{n} \frac{1}{2} \tilde{A}_{i} \lambda_{j}^{n-i} & \sum_{i=0}^{n} \tilde{\delta}_{i} \lambda_{j}^{n-i} \\
\sum_{i=0}^{n} \tilde{\delta}_{i} \lambda_{j}^{n-i} & -\sum_{i=0}^{n} \tilde{\rho}_{i} \lambda_{j}^{n-i} & 0
\end{array}\right)\left(\begin{array}{l}
\phi_{1 j} \\
\phi_{2 j} \\
\phi_{3 j}
\end{array}\right), \\
\left(\begin{array}{c}
\psi_{1 j} \\
\psi_{2 j} \\
\psi_{3 j}
\end{array}\right)_{t_{n}}=\left(\begin{array}{ccc}
-\sum_{i=0}^{n} \frac{1}{2} \tilde{A}_{i} \lambda_{j}^{n-i} & -\sum_{i=0}^{n} \tilde{C}_{i} \lambda_{j}^{n-i} & \sum_{i=0}^{n} \tilde{\delta}_{i} \lambda_{j}^{n-i} \\
-\sum_{i=0}^{n} \tilde{B}_{i} \lambda_{j}^{n-i} & \sum_{i=0}^{n} \frac{1}{2} \tilde{A}_{i} \lambda_{j}^{n-i} & -\sum_{i=0}^{n} \tilde{\rho}_{i} \lambda_{j}^{n-i} \\
-\sum_{i=0}^{n} \tilde{\rho}_{i} \lambda_{j}^{n-i} & -\sum_{i=0}^{n} \tilde{\delta}_{i} \lambda_{j}^{n-i} & 0
\end{array}\right)\left(\begin{array}{l}
\psi_{1 j} \\
\psi_{2 j} \\
\psi_{3 j}
\end{array}\right), \\
\left\{\begin{array}{c}
\Phi_{1, x}=\frac{1}{2} \Lambda \Phi_{1}+\frac{1}{2}\left\langle\Psi_{2}, \Phi_{1}\right\rangle \Phi_{2}-\frac{1}{4}\left(\left\langle\Psi_{2}, \Phi_{3}\right\rangle-\left\langle\Psi_{3}, \Phi_{1}\right\rangle\right) \Phi_{3}, \\
\Phi_{2, x}=\frac{1}{2}\left\langle\Psi_{1}, \Phi_{2}\right\rangle \Phi_{1}-\frac{1}{2} \Lambda \Phi_{2}+\frac{1}{4}\left(\left\langle\Psi_{1}, \Phi_{3}\right\rangle+\left\langle\Psi_{3}, \Phi_{2}\right\rangle\right) \Phi_{3}, \\
\Phi_{3, x}=\frac{1}{4}\left(\left\langle\Psi_{1}, \Phi_{3}\right\rangle+\left\langle\Psi_{3}, \Phi_{2}\right\rangle\right) \Phi_{1}+\frac{1}{4}\left(\left\langle\Psi_{2}, \Phi_{3}\right\rangle-\left\langle\Psi_{3}, \Phi_{1}\right\rangle\right) \Phi_{2}, \\
\Psi_{1, x}=-\frac{1}{2} \Lambda \Psi_{1}-\frac{1}{2}\left\langle\Psi_{1}, \Phi_{2}\right\rangle \Psi_{2}+\frac{1}{4}\left(\left\langle\Psi_{1}, \Phi_{3}\right\rangle+\left\langle\Psi_{3}, \Phi_{2}\right\rangle\right) \Psi_{3}, \\
\Psi_{2, x}=-\frac{1}{2}\left\langle\Psi_{2}, \Phi_{1}\right\rangle \Psi_{1}+\frac{1}{2} \Lambda \Psi_{2}+\frac{1}{4}\left(\left\langle\Psi_{2}, \Phi_{3}\right\rangle-\left\langle\Psi_{3}, \Phi_{1}\right\rangle\right) \Psi_{3}, \\
\Psi_{3, x}=\frac{1}{4}\left(\left\langle\Psi_{2}, \Phi_{3}\right\rangle-\left\langle\Psi_{3}, \Phi_{1}\right\rangle\right) \Psi_{1}-\frac{1}{4}\left(\left\langle\Psi_{1}, \Phi_{3}\right\rangle+\left\langle\Psi_{3}, \Phi_{2}\right\rangle\right) \Psi_{2},
\end{array}\right.
\end{gathered}
$$


When $n=2$, the system Equation (26) is

$$
\left\{\begin{aligned}
\Phi_{1, t_{2}}= & \left(\frac{1}{2} \Lambda^{2}-\frac{1}{4} \tilde{q}^{2}+\frac{1}{4} \tilde{r}^{2}-2 \tilde{\alpha} \tilde{\beta}\right) \Phi_{1}+\left(\frac{1}{2}(\tilde{q}+\tilde{r}) \Lambda+\frac{1}{2}\left(\tilde{q}_{x}+\tilde{r}_{x}\right)\right) \Phi_{2} \\
& +\left(\tilde{\alpha} \Lambda+2 \tilde{\alpha}_{x}\right) \Phi_{3}, \\
\Phi_{2, t_{2}}= & \left(\frac{1}{2}(\tilde{q}-\tilde{r}) \Lambda-\frac{1}{2}\left(\tilde{q}_{x}-\tilde{r}_{x}\right)\right) \Phi_{1}-\left(\frac{1}{2} \Lambda^{2}-\frac{1}{4} \tilde{q}^{2}+\frac{1}{4} \tilde{r}^{2}-2 \tilde{\alpha} \tilde{\beta}\right) \Phi_{2} \\
& +\left(\tilde{\beta} \Lambda-2 \tilde{\beta}_{x}\right) \Phi_{3}, \\
& \Phi_{3, t_{2}}=\left(\tilde{\beta} \Lambda-2 \tilde{\beta}_{x}\right) \Phi_{1}-\left(\tilde{\alpha} \Lambda+2 \tilde{\alpha}_{x}\right) \Phi_{2}, \\
\Psi_{1, t_{2}=} & \left(-\frac{1}{2} \Lambda^{2}+\frac{1}{4} \tilde{q}^{2}-\frac{1}{4} \tilde{r}^{2}+2 \tilde{\alpha} \tilde{\beta}\right) \Psi_{1}-\left(\frac{1}{2}(\tilde{q}-\tilde{r}) \Lambda-\frac{1}{2}\left(\tilde{q}_{x}-\tilde{r}_{x}\right)\right) \Psi_{2} \\
& +\left(\tilde{\beta} \Lambda-2 \tilde{\beta}_{x}\right) \Psi_{3}, \\
\Psi_{2, t_{2}=} & -\left(\frac{1}{2}(\tilde{q}+\tilde{r}) \Lambda+\frac{1}{2}\left(\tilde{q}_{x}+\tilde{r}_{x}\right)\right) \Psi_{1}+\left(\frac{1}{2} \Lambda^{2}-\frac{1}{4} \tilde{q}^{2}+\frac{1}{4} \tilde{r}^{2}-2 \tilde{\alpha} \tilde{\beta}\right) \Psi_{2} \\
& -\left(\tilde{\alpha} \Lambda+2 \tilde{\alpha}_{x}\right) \Psi_{3}, \\
& \Psi_{3, t_{2}}=\left(\tilde{\beta} \Lambda-2 \tilde{\beta}_{x}\right) \Psi_{1}-\left(\tilde{\alpha} \Lambda+2 \tilde{\alpha}_{x}\right) \Psi_{2},
\end{aligned}\right.
$$

where $\tilde{q}, \tilde{r}, \tilde{\alpha}, \tilde{\beta}$ denote the functions $q, r, \alpha, \beta$ defined by the explicit constraint Equation (24), and $\tilde{q}_{x}, \tilde{r}_{x}, \tilde{\alpha}_{x}, \tilde{\beta}_{x}$ are given by

$$
\left\{\begin{array}{c}
\tilde{q}_{x}=\frac{1}{2}\left\langle\Lambda \Psi_{2}, \Phi_{1}\right\rangle-\frac{1}{2}\left\langle\Lambda \Psi_{1}, \Phi_{2}\right\rangle+\frac{1}{4}\left(\left\langle\Psi_{1}, \Phi_{2}\right\rangle-\left\langle\Psi_{2}, \Phi_{1}\right\rangle\right)\left(\left\langle\Psi_{1}, \Phi_{1}\right\rangle-\left\langle\Psi_{2}, \Phi_{2}\right\rangle\right), \\
\tilde{r}_{x}=\frac{1}{2}\left\langle\Lambda \Psi_{2}, \Phi_{1}\right\rangle+\frac{1}{2}\left\langle\Lambda \Psi_{1}, \Phi_{2}\right\rangle-\frac{1}{4}\left(\left\langle\Psi_{1}, \Phi_{2}\right\rangle+\left\langle\Psi_{2}, \Phi_{1}\right\rangle\right)\left(\left\langle\Psi_{1}, \Phi_{1}\right\rangle-\left\langle\Psi_{2}, \Phi_{2}\right\rangle\right), \\
\tilde{\alpha}_{x}=-\frac{1}{8}\left\langle\Lambda \Psi_{2}, \Phi_{3}\right\rangle+\frac{1}{8}\left\langle\Lambda \Psi_{3}, \Phi_{1}\right\rangle+\frac{1}{16}\left(\left\langle\Psi_{1}, \Phi_{1}\right\rangle-\left\langle\Psi_{2}, \Phi_{2}\right\rangle\right)\left(\left\langle\Psi_{2}, \Phi_{3}\right\rangle-\left\langle\Psi_{3}, \Phi_{1}\right\rangle\right), \\
\tilde{\beta}_{x}=-\frac{1}{8}\left\langle\Lambda \Psi_{1}, \Phi_{3}\right\rangle-\frac{1}{8}\left\langle\Lambda \Psi_{3}, \Phi_{2}\right\rangle+\frac{1}{16}\left(\left\langle\Psi_{1}, \Phi_{1}\right\rangle-\left\langle\Psi_{2}, \Phi_{2}\right\rangle\right)\left(\left\langle\Psi_{1}, \Phi_{3}\right\rangle+\left\langle\Psi_{3}, \Phi_{2}\right\rangle\right) .
\end{array}\right.
$$

which are computed through using the spatial constrained flow Equation (27).

The system Equation (29) can be represented as the following super Hamiltonian form:

$$
\left\{\begin{array}{c}
\Phi_{1, t_{2}}=\frac{\partial H_{2}}{\partial \Psi_{1}}, \Phi_{2, t_{2}}=\frac{\partial H_{2}}{\partial \Psi_{2}}, \Phi_{3, t_{2}}=\frac{\partial H_{2}}{\partial \Psi_{3}} \\
\Psi_{1, t_{2}}=-\frac{\partial H_{2}}{\partial \Phi_{1}}, \Psi_{2, t_{2}}=-\frac{\partial H_{2}}{\partial \Phi_{2}}, \Psi_{3, t_{2}}=\frac{\partial H_{2}}{\partial \Phi_{3}} .
\end{array}\right.
$$

where

$$
\begin{aligned}
H_{2}= & \frac{1}{2}\left\langle\Lambda^{2} \Psi_{1}, \Phi_{1}\right\rangle-\frac{1}{2}\left\langle\Lambda^{2} \Psi_{2}, \Phi_{2}\right\rangle \\
& -\frac{1}{4}\left\langle\Psi_{2}, \Phi_{1}\right\rangle\left\langle\Psi_{1}, \Phi_{2}\right\rangle\left(\left\langle\Psi_{1}, \Phi_{1}\right\rangle-\left\langle\Psi_{2}, \Phi_{2}\right\rangle\right) \\
& +\frac{1}{8}\left(\left\langle\Psi_{2}, \Phi_{3}\right\rangle-\left\langle\Psi_{3}, \Phi_{1}\right\rangle\right)\left(\left\langle\Psi_{1}, \Phi_{3}\right\rangle+\left\langle\Psi_{3}, \Phi_{2}\right\rangle\right) \\
& \times\left(\left\langle\Psi_{1}, \Phi_{1}\right\rangle-\left\langle\Psi_{2}, \Phi_{2}\right\rangle\right) \\
& +\frac{1}{2}\left\langle\Lambda \Psi_{2}, \Phi_{1}\right\rangle\left\langle\Psi_{1}, \Phi_{2}\right\rangle+\frac{1}{2}\left\langle\Psi_{2}, \Phi_{1}\right\rangle\left\langle\Lambda \Psi_{1}, \Phi_{2}\right\rangle \\
& -\frac{1}{4}\left(\left\langle\Lambda \Psi_{2}, \Phi_{3}\right\rangle-\left\langle\Lambda \Psi_{3}, \Phi_{1}\right\rangle\right)\left(\left\langle\Psi_{1}, \Phi_{3}\right\rangle+\left\langle\Psi_{3}, \Phi_{2}\right\rangle\right) \\
& -\frac{1}{4}\left(\left\langle\Psi_{2}, \Phi_{3}\right\rangle-\left\langle\Psi_{3}, \Phi_{1}\right\rangle\right)\left(\left\langle\Lambda \Psi_{1}, \Phi_{3}\right\rangle+\left\langle\Lambda \Psi_{3}, \Phi_{2}\right\rangle\right) .
\end{aligned}
$$

In what follows, we want to prove that Equation (25) is a completely integrable Hamiltonian system in the Liouville sense. Furthermore, we shall prove that Equation (26) is also completely integrable under the contron of system Equation (25).
In addition, the characteristic property Equation (20) and the recurrence relations Equation (5) ensure that

$$
\begin{cases}\tilde{A}_{i+1}=\frac{1}{2}\left(\left\langle\Lambda^{i} \Psi_{1}, \Phi_{1}\right\rangle-\left\langle\Lambda^{i} \Psi_{1}, \Phi_{1}\right\rangle\right), & i \geq 0, \\ \tilde{B}_{i+1}=\frac{1}{2}\left\langle\Lambda^{i} \Psi_{2}, \Phi_{1}\right\rangle, & i \geq 0, \\ \tilde{C}_{i+1}=\frac{1}{2}\left\langle\Lambda^{i} \Psi_{1}, \Phi_{2}\right\rangle, & i \geq 0, \\ \tilde{\rho}_{i+1}=-\frac{1}{4}\left(\left\langle\Lambda^{i} \Psi_{2}, \Phi_{3}\right\rangle-\left\langle\Lambda^{i} \Psi_{3}, \Phi_{1}\right\rangle\right), & i \geq 0, \\ \tilde{\delta}_{i+1}=\frac{1}{4}\left(\left\langle\Lambda^{i} \Psi_{1}, \Phi_{3}\right\rangle+\left\langle\Lambda^{i} \Psi_{3}, \Phi_{2}\right\rangle\right), & i \geq 0 .\end{cases}
$$

Then the co-adjoint representation equation $\tilde{V}_{x}=[\tilde{U}, \tilde{V}]$ remains true. Furthermore, we know that $\tilde{V}_{x}^{2}=\left[\tilde{U}, \tilde{V}^{2}\right]$ is also true. Let

$$
F=\operatorname{Str} \tilde{V}^{2} .
$$

Then it is easy to find that $F_{x}=0$. That is easy to see, $F$ is a generating function of integrals of motion for the system Equation (25) or Equation (27). Due to

$$
F=\sum_{n \geq 0} F_{n} \lambda^{-n},
$$

we obtain the following formulas of integrals of motion:

$$
\left\{\begin{array}{c}
F_{0}=\frac{1}{2} \tilde{A}_{0}^{2}, F_{1}=\tilde{A}_{0} \tilde{A}_{1}, \\
F_{n}=\tilde{A}_{0} \tilde{A}_{n}+\sum_{i=1}^{n-1}\left(\frac{1}{2} \tilde{A}_{i} \tilde{A}_{n-i}+2 \tilde{B}_{i} \tilde{C}_{n-i}+4 \tilde{\rho}_{i} \tilde{\delta}_{n-i}\right), i \geq 2
\end{array}\right.
$$


Substituting Equation (32) into the above formulas of motion, we obtain the following expression of $F_{m}(m \geq 0)$ :

$$
\begin{aligned}
F_{0}= & \frac{1}{2}, F_{1}=\frac{1}{2}\left(\left\langle\Psi_{1}, \Phi_{1}\right\rangle-\left\langle\Psi_{2}, \Phi_{2}\right\rangle\right) \\
F_{2}= & \frac{1}{2}\left(\left\langle\Lambda \Psi_{1}, \Phi_{1}\right\rangle-\left\langle\Lambda \Psi_{2}, \Phi_{2}\right\rangle\right) \\
& +\frac{1}{2}\left\langle\Psi_{2}, \Phi_{1}\right\rangle\left\langle\Psi_{1}, \Phi_{2}\right\rangle \\
& -\frac{1}{4}\left(\left\langle\Psi_{2}, \Phi_{3}\right\rangle-\left\langle\Psi_{3}, \Phi_{1}\right\rangle\right)\left(\left\langle\Psi_{1}, \Phi_{3}\right\rangle+\left\langle\Psi_{3}, \Phi_{2}\right\rangle\right) \\
F_{n}= & \frac{1}{2}\left(\left\langle\Lambda^{n-1} \Psi_{1}, \Phi_{1}\right\rangle-\left\langle\Lambda^{n-1} \Psi_{2}, \Phi_{2}\right\rangle\right) \\
+ & \sum_{i=1}^{n-1} \frac{1}{2}\left\langle\Lambda^{i-1} \Psi_{2}, \Phi_{1}\right\rangle\left\langle\Lambda^{n-i-1} \Psi_{1}, \Phi_{2}\right\rangle \\
+ & \sum_{i=1}^{n-1} \frac{1}{8}\left(\left\langle\Lambda^{i-1} \Psi_{1}, \Phi_{1}\right\rangle-\left\langle\Lambda^{i-1} \Psi_{2}, \Phi_{2}\right\rangle\right) \\
& \cdot\left(\left\langle\Lambda^{n-i-1} \Psi_{1}, \Phi_{1}\right\rangle-\left\langle\Lambda^{n-i-1} \Psi_{2}, \Phi_{2}\right\rangle\right) \\
& -\sum_{i=1}^{n-1} \frac{1}{4}\left(\left\langle\Lambda^{i-1} \Psi_{2}, \Phi_{3}\right\rangle-\left\langle\Lambda^{i-1} \Psi_{3}, \Phi_{1}\right\rangle\right) \\
& \cdot\left(\left\langle\Lambda^{n-i-1} \Psi_{1}, \Phi_{3}\right\rangle+\left\langle\Lambda^{n-i-1} \Psi_{3}, \Phi_{2}\right\rangle\right)
\end{aligned}
$$

$n \geq 2$.

On the other hand, let us consider the temporal part of the nonlinearized system Equation (26). Making use of Equation (32) and Equation (36), the system Equation (26) can be written as the following super Hamiltonian form:

$$
\left\{\begin{array}{c}
\Phi_{1, t_{n}}=\frac{\partial F_{n+1}}{\partial \Psi_{1}}, \Phi_{2, t_{n}}=\frac{\partial F_{n+1}}{\partial \Psi_{2}}, \Phi_{3, t_{n}}=\frac{\partial F_{n+1}}{\partial \Psi_{3}}, \\
\Psi_{1, t_{n}}=-\frac{\partial F_{n+1}}{\partial \Phi_{1}}, \Psi_{2, t_{n}}=-\frac{\partial F_{n+1}}{\partial \Phi_{2}}, \Psi_{3, t_{n}}=\frac{\partial F_{n+1}}{\partial \Phi_{3}} .
\end{array}\right.
$$

This can be checked pretty easily. For example, we can show one equality in the above system as follows:

$$
\begin{aligned}
\Phi_{1, t_{n}}= & \sum_{i=0}^{n} \frac{1}{2} A_{i} \Lambda^{n-i} \Phi_{1}+\sum_{i=0}^{n} B_{i} \Lambda^{n-i} \Phi_{2}+\sum_{i=0}^{n} \rho_{i} \Lambda^{n-i} \Phi_{3} \\
= & \frac{1}{2} \Lambda^{n} \Phi_{1}+\frac{1}{4} \sum_{i=1}^{n}\left(\left\langle\Lambda^{i-1} \Psi_{1}, \Phi_{1}\right\rangle-\left\langle\Lambda^{i-1} \Psi_{2}, \Phi_{2}\right\rangle\right) \Lambda^{n-i} \Phi_{1} \\
& +\frac{1}{2} \sum_{i=1}^{n}\left\langle\Lambda^{i-1} \Psi_{2}, \Phi_{1}\right\rangle \Lambda^{n-i} \Phi_{2} \\
& -\frac{1}{4} \sum_{i=1}^{n}\left(\left\langle\Lambda^{i-1} \Psi_{2}, \Phi_{3}\right\rangle-\left\langle\Lambda^{i-1} \Psi_{3}, \Phi_{1}\right\rangle\right) \Lambda^{n-i} \Phi_{3} \\
= & \frac{\partial F_{n+1}}{\partial \Psi_{1}} .
\end{aligned}
$$

In order to show the Liouville integrability for the constrained flows Equation (25) and Equation (26), we need to prove the commutative propertity of motion $\left\{F_{m}\right\}_{m \geq 0}$, under the corresponding Poission bracket

$$
\{F, G\}=\sum_{i=1}^{3} \sum_{j=1}^{N}\left(\frac{\partial F}{\partial \phi_{i j}} \frac{\partial G}{\partial \psi_{i j}}-(-1)^{p\left(\phi_{i j}\right) p\left(\psi_{i j}\right)} \frac{\partial F}{\partial \psi_{i j}} \frac{\partial G}{\partial \phi_{i j}}\right) \text {. }
$$

At this time, we still have an equality $\tilde{V}_{t_{n}}=\left[\tilde{V}^{(n)}, \tilde{V}\right]$, and after a similar discussion, we know that $F$ is also a generating function of integrals of motion for Equation (26). Hence $\left\{F_{m}\right\}_{m \geq 0}$ are integrals of motion for the system Equation (26) or Equation (37), which implies

$$
\left\{F_{m+1}, F_{n+1}\right\}=\frac{\partial}{\partial t_{n}} F_{m+1}=0, m, n \geq 0 .
$$

The above equality Equation (40) shows that $\left\{F_{m}\right\}_{m \geq 0}$ are in involution in pair under the Poission bracket Equation (39).

In addition, similar to the method in [16], we know that

$$
f_{k}=\psi_{1 k} \phi_{1 k}+\psi_{2 k} \phi_{2 k}+\psi_{3 k} \phi_{3 k}, 1 \leq k \leq N .
$$

are integrals of motion for Equation (25) and Equation (26). It is not difficult to verify that the $3 N$ functions $\left\{F_{m}\right\}_{m=1}^{2 N}$ and $\left\{f_{k}\right\}_{k=1}^{N}$ are involution in pair. Similar to the methods in $[10,16,17]$, we can verify that the $3 N$ functions $\left\{F_{m}\right\}_{m=1}^{2 N}$ and $\left\{f_{k}\right\}_{k=1}^{N}$ are functionally independent over some region of the super symmetry manifold $\mathbb{R}^{4 N \mid 2 N}$. Now, all of above analysis gives the following theorem.

Theorem 1 Both the spatial and temporal flows Equation (25) and Equation (26) are Liouville integrable super Hamiltonian systems defined on the super symmetry manifold $\mathbb{R}^{4 N \mid 2 N}$, which possess $3 N$ functionally independent and involutive integrals $\left\{F_{m}\right\}_{m=1}^{2 N}$ and $\left\{f_{k}\right\}_{k=1}^{N}$ defined by Equation (36) and Equation (41).

\section{Acknowledgements}

This work was supported by the Natural Science Foundation of China ( No. 61072147), the Science and Technology Key Research Foundation of Provincial Education Department of Henan (No. 12A110017), the Youth Research Foundation of Shangqiu Normal University (No. 2011QN12).

\section{REFERENCES}

[1] Y. Cheng and Y. S. Li, "The Constraint of The Kadomtsev-Petviashvili Equation and Its Special Solutions," Physics Letters A, Vol. 157, No. 1, 1991, pp. 22-26. doi: 10.1016/0375-9601(91)90403-U

[2] Y. Cheng, "Constraints of the Kadomtsev-Petviashvili Hierarchy," Journal of Mathematical Physics, Vol. 33, No. 11, 1992, pp. 3774-3782. doi: 10.1063/1.529875

[3] W. X. Ma and W Strampp, “An Explicit Symmetry Constraint for the Lax Pairs of AKNS Systems," Physics Letters A, Vol. 185, No. 3, 1994, pp. 277-286. 


\section{doi: 10.1016/0375-9601(94)90616-5}

[4] W. X. Ma, "New Finite-Dimensional Integrable Systems by Symmetry Constraint of the KdV Equations," Journal of the Physical Society of Japan, Vol. 64, No. 4, 1995, pp. 1085-1091. doi: 10.1143/JPSJ.64.1085

[5] Y. B. Zeng and Y. S. Li, "The Constraints of Potentials and the Finite-Dimensional Integrable Systems,” Journal of Mathematical Physics, Vol. 30, No. 8, 1989, pp.1679-1689. doi:10.1063/1.528253

[6] C. W. Cao and X. G. Geng, “A Monconfocal Generator of Involutive Systems and Three Associated Soliton Hierarchies," Journal of Mathematical Physics, Vol. 2, No. 9, 1991, pp. 2323-2328. doi:10.1063/1.529156

[7] W. X. Ma, "Symmetry Constraint of MKdV Equations by Binary Nonlinearization,” Physica A, Vol. 219, No. 3-4, 1995, pp. 467-481. doi:10.1016/0378-4371(95)00161-Y

[8] W. X. Ma and R. G. Zhou, "Adjoint Symmetry Constraints Leading to Binaary Nonlinearization," Journal of Nonlinear Mathematical Physics, Vol. 9, No. Suppl. 1, 2002, pp. 106-126.

[9] W. X. Ma, "Binary Bonlinearization for the Dirac Systems," Chinese Annals of Mathematics, Series B, Vol. 18, No. 1, 1997, pp. 79-88.

[10] J. S. He, J. Yu, Y. Cheng and R. G. Zhou, "Binary Bon linearization of the Super AKNS System,” Modern Physics Letters B, Vol. 22, No. 4, 2008, pp. 275-288. doi:10.1142/S0217984908014778

[11] J. Yu, J. W. Han and J. S. He, "Binary Nonlinearization of the Super AKNS System under an Implicit Symmetry constraint," Journal of Physics A: Mathematical and Theoretical, Vol. 42, No. 46, 2009, 465201. doi:10.1088/1751-8113/42/46/465201

[12] J. Yu, J. S. He, W. X. Ma and Y. Cheng, “The Bargmann Symmetry Constraint and Binary Nonlinearization of the Super Dirac System," Chinese Annals of Mathematics, Series B, Vol. 31, No. 3, 2010, pp. 361-372. doi:10.1007/s11401-009-0032-6

[13] H. H. Dong and X. Z. Wang, "Lie Algebra and Lie Super Algebra for the Integrable Couplings of NLS-MKdV Hierarchy," Communications in Nonlinear Science and Numeical Simulation, Vol. 14, No. 12, 2009, pp. 4071-4077. doi:10.1016/j.cnsns.2009.03.010

[14] W. X. Ma, J. S. He and Z. Y. Qin, “A Supertrace Identity and Its Applications to Super Integrable Systems," Journal of Mathematical Physics, Vol. 49, No. 3, 2008, 033511. doi:10.1063/1.2897036

[15] X. B. Hu, "An Approach to Generate Superextensions of Integrable Systems,” Journal of Physics A: Mathematical and General, Vol. 30, No. 2, 1997, pp. 619-632. doi:10.1088/0305-4470/30/2/023

[16] W. X. Ma, B. Fuchssteiner and W. Oevel, “A $3 \times 3$ Matrix Spectral Problem for AKNS Hierarchy and Its Binary Nonlinearization,” Physica A, Vol. 233, No. 1-2, 1996, pp. 331-354. doi: 10.1016/S0378-4371(96)00225-7

[17] W. X. Ma and Z. X. Zhou, "Binary Symmetry Constraints of $\mathrm{N}$-wave Intersection Equations in 1+1 and 2+1 Dimensions,” Journal of Mathematical Physics, Vol. 42, No. 9, 2001, pp. 4345-4382. doi:10.1063/1.1388898 